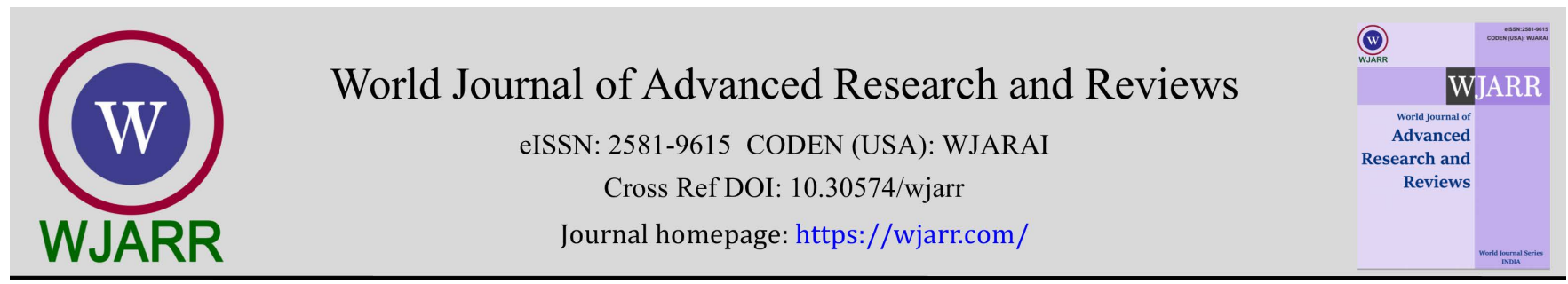

(RESEARCh ARTiCle)

Check for updates

\title{
Diurnal glycemic pattern on inclusion of sucrose in daily meals in type 2 diabetes: Reflection of antecedent glycemic control
}

\author{
Udaya M Kabadi * \\ Central Iowa Veteran's Affairs System, Des Moines Iowa and University of Iowa, Iowa City Iowa.
}

World Journal of Advanced Research and Reviews, 2021, 12(02), 197-201

Publication history: Received on 01 October 2021; revised on 07 November 2021; accepted on 09 November 2021

Article DOI: https://doi.org/10.30574/wjarr.2021.12.2.0576

\begin{abstract}
Background: Inclusion of sucrose in diabetic diets is not recommended in subjects with diabetes since effects of such diets on glycemic indices are not established. Some studies have documented lapse in metabolic control on consumption of these diets type 2 diabetes. However, most studies examined plasma glucose only for a few hours after ingestion of a single meal containing sucrose after an overnight fast whereas others recommended increasing insulin dose prior to the meal. None of these studies examined influence of inclusion of sucrose in daily meals in subjects with new onset diabetes at diagnosis and again after achieving desirable glycemic control.
\end{abstract}

Objective : Study was conducted to assess glycemic responses to ingestion of all meals containing sucrose constituting $50 \%$ of carbohydrate calories.

Methods: 12 subjects with new onset type 2 diabetes participated. They were administered the following isocaloric diets for 4 days each prior to and after achieving desirable glycemic control; Diet 1- Diabetic diet recommended by American Diabetes Association (ADA), diet 2- test diet containing sucrose, diet 3- ADA diet. Glycemic control was assessed by diurnal glycemia (average of pre-prandial, postprandial and bedtime blood glucose), fasting plasma glucose, HbA1c and fructosamine on $4^{\text {th }}$ day of each dietary period.

Results: All glycemic indices deteriorated after consumption of sucrose containing meals prior to initiation of treatment and remained worsened on return to ingestion of ADA diet. Glycemic responses after all meals improved markedly on achieving desirable glycemic control. Moreover, glycemic indices remained unaltered on consumption of sucrose containing meals after attaining and maintaining desirable glycemic control.

Conclusion: Diurnal glycemic responses deteriorate on ingestion of daily meals containing sucrose in subjects with new onset uncontrolled type 2 diabetes. In contrast, diurnal glycemic pattern is unaltered following consumption of daily meals containing sucrose after attaining and maintaining desirable glycemic control.

Keywords: Diurnal Glycemia; Sucrose; Hemoglobin A1c; Fructosamine

\section{Introduction}

Rise in plasma glucose follows ingestion of 75 g. glucose during glucose tolerance tests in subjects with diabetes as well as normal subjects [1-3]. However, the rise in subjects with new onset diabetes is markedly greater as compared to normal subjects [1-3]. We have also documented marked improvement in glucose responses during OGTT in subjects with type 2 diabetes on attaining desirable glycemic control [1]. Inclusion of sucrose in a meal is not recommended

\footnotetext{
${ }^{*}$ Corresponding author: Udaya M Kabadi

Central Iowa Veteran's Affairs System, Des Moines Iowa and University of Iowa, Iowa City Iowa. 
probably because of the concern about a marked rise in postprandial glucose level as noted during OGTT [4]. However, ingestion of glucose or sucrose alone during Oral Glucose tolerance test is not comparable to inclusion of sucrose in a mixed meal as the meal consists of all food constituents as well as fiber. Moreover, the influence of attaining desirable glycemic control on diurnal glucose pattern following inclusion of sucrose in all daily meals for several days is not reported. Therefore, this study was conducted to assess diurnal glycemic responses following ingestion of all three main meals containing sucrose providing $50 \%$ of total carbohydrate content in subjects with new onset type 2 diabetes prior to and following achieving desirable glycemic control.

\section{Subjects and methods}

The study was approved by Research and development committee as well as human studies subcommittee at a major academic medical center. 12 subjects, 7 men and 5 women with ages 41-67 years (mean age, 55 \pm 8 ) with newly diagnosed type 2 diabetes mellitus participated after signing informed consent. The dietary study procedures were conducted prior to initiation of treatment and again after attaining and maintaining desirable glycemic control (HbA1c $<7.0 \%)$ for 3 months using metformin alone or in combination with Glimepiride (Table1). The study procedure consisted of administration of 3 isocaloric diets for 4 days. Diet 1- diet recommended by American Diabetes Association (ADA); Diet 2 - test diet containing sucrose contributing 50\% of the total carbohydrate content to each meal; Diet 3-ADA diet. Diurnal glycemic control was assessed by 7 point glucose testing (pre-prandial and 2 hour postprandial with each meal and bedtime) on the last day of each diet protocol. Glucose testing was performed by blood obtained by finger stick using 'Freestyle lite' glucose meter (Abbot Laboratory, Abbot Park, Illinois, USA ) Fasting plasma glucose, fructosamine and HbA1c were determined on the last day of each diet period as well. These tests were performed by the local laboratory using established assay methods. Coefficients of variation for all determinations were established to be between 9.0 to $15 \%$. Comparisons were conducted between these glycemic indices following dietary protocols prior to as well as on attaining and maintaining desirable glycemic control. Comparisons were also conducted between various diet protocols 1) prior to initiation of treatment as well as 2) after attaining desirable glycemic control. Statistical analyses were performed using Student's ' $t$ ' test and analysis of variance. All values are reported as Mean \pm Standard Error of Mean (SEM).

Table 1 Mean pre-prandial (PreG)†, 2 hour postprandial (PostG)†, fasting plasma Glucose(FPG), average diurnal blood glucose (MPG) F, HbA1c and Fructosamine (Fruct) concentrations prior to (Pre Rx) and after (Post Rx) attaining and maintaining desirable glycemic control (HbA1c $\leq 7 \%)$ in 12 subjects in new onset type 2 diabetes mellitus

\begin{tabular}{|l|l|l|l|l|l|l|}
\hline DIET & $\begin{array}{l}\text { PreG } \\
\mathbf{m g} / \mathbf{d l}\end{array}$ & $\begin{array}{l}\text { PostG } \\
\mathbf{m g} / \mathbf{d l}\end{array}$ & $\begin{array}{l}\text { FPG } \\
\mathbf{~ m g / d l}\end{array}$ & $\begin{array}{l}\text { MPG } \\
\mathbf{m g} / \mathbf{d l}\end{array}$ & $\begin{array}{l}\text { HbA1c } \\
\mathbf{\%}\end{array}$ & $\begin{array}{l}\text { Fruct } \\
\text { umol/1 }\end{array}$ \\
\hline Pre Rx & $203 \pm 10$ & $265 \pm 17$ & $206 \pm 13$ & $236 \pm 15$ & $9.3 \pm 0.3$ & $334 \pm 18$ \\
\hline Post Rx & $113 \pm 8 *$ & $153 \pm 16 *$ & $125 \pm 9 *$ & $133 \pm 8 *$ & $6.7 \pm 0.2 *$ & $264 \pm 14 *$ \\
\hline
\end{tabular}

\section{Results}

All glycemic indices as expressed by pre-prandial, 2 hour postprandial and mean diurnal blood glucose levels as well as fasting plasma glucose, HbA1c and fructosamine concentrations improved significantly on attaining and maintaining desirable HbA1c levels following treatment in all subjects (Table1). Prior to initiation of treatment, all the same glycemic indices rose significantly following consumption of sucrose containing diet (diet 2) when compared with ADA diet (diet1). Moreover, the worse diurnal glycemic indices observed after consumption of diet 2 for 4 days persisted despite return to ADA diet for 4 days (Table 2). In contrast, on attaining and maintaining desirable glycemic control, all diurnal glycemic indices were not significantly altered following ingestion of sucrose containing diet 2 when compared with consumption of ADA diets 1 and 3 (Table 3). 
Table 2 Mean pre-prandial (PreG)†, 2 hour postprandial (PostG)†,, fasting plasma Glucose(FPG), Mean diurnal blood glucose (MPG) $\mathscr{F}$, HbA1c and Fructosamine (Fruct) concentrations on 4th day of study diets in 12 subjects with new onset type 2 diabetes prior to initiation of treatment

\begin{tabular}{|c|c|c|c|c|c|c|}
\hline DIET & $\begin{array}{c}\text { PreG } \\
\mathbf{m g} / \mathbf{d l}\end{array}$ & $\begin{array}{c}\text { PostG } \\
\mathbf{m g} / \mathbf{d l}\end{array}$ & $\begin{array}{c}\text { FPG } \\
\mathbf{m g} / \mathbf{d l}\end{array}$ & $\begin{array}{c}\text { MPG } \\
\mathbf{~ m g / d l}\end{array}$ & $\begin{array}{c}\text { HbA1c } \\
\%\end{array}$ & $\begin{array}{c}\text { Fruct } \\
\text { umol/l }\end{array}$ \\
\hline 1 & $203 \pm 10$ & $265 \pm 17$ & $206 \pm 10$ & $236 \pm 15$ & $9.3 \pm 0.2$ & $334 \pm 18$ \\
\hline 2 & $236 \pm 15^{*}$ & $309 \pm 20 *$ & $232 \pm 13^{*}$ & $271 \pm 18 *$ & $9.8 \pm 0.4 *$ & $377 \pm 21 *$ \\
\hline 3 & $235 \pm 16^{*}$ & $314 \pm 21 *$ & $228 \pm 11 *$ & $275 \pm 17 *$ & $9.6 \pm 0.3 *$ & $381 \pm 20 *$ \\
\hline
\end{tabular}

Table 3 Mean pre-prandial (PreG)†, 2 hour postprandial (PostG)†, fasting plasma Glucose(FPG), average diurnal blood glucose (MPG) $\mathscr{F}, \mathrm{HbA1c}$ and Fructosamine We were (Fruct) concentrations on 4th day of study diets in 12 subjects after attaining and maintenance of desirable glycemic control ( A1c $<7.0 \%$ )

\begin{tabular}{|c|c|c|c|c|c|c|}
\hline DIET & $\begin{array}{c}\text { PreG } \\
\mathbf{m g} / \mathbf{d l}\end{array}$ & $\begin{array}{c}\text { PostG } \\
\mathbf{m g} / \mathbf{d l}\end{array}$ & $\begin{array}{c}\text { FPG } \\
\mathbf{m g} / \mathbf{d l}\end{array}$ & $\begin{array}{c}\text { MPG } \\
\mathbf{m g} / \mathbf{d l}\end{array}$ & $\begin{array}{c}\text { HbA1c } \\
\mathbf{\%}\end{array}$ & $\begin{array}{c}\text { Fruct } \\
\mathbf{u m o l} / \mathbf{l}\end{array}$ \\
\hline 1 & $113 \pm 8$ & $153 \pm 16$ & $125 \pm 9$ & $133 \pm 8$ & $6.7 \pm 0.2$ & $264 \pm 14$ \\
\hline 2 & $116 \pm 10$ & $151 \pm 18$ & $131 \pm 10$ & $135 \pm 9$ & $6.8 \pm 0.2$ & $267 \pm 16$ \\
\hline 3 & $115 \pm 8$ & $144 \pm 14$ & $129 \pm 8$ & $129 \pm 7$ & $6.6 \pm 0.2$ & $261 \pm 11$ \\
\hline
\end{tabular}

† Mean of 3 values on 4 th day of study diets; $\mathscr{F}$ Mean of 7 values on 4 th day of study diets

\section{Discussion}

Several studies have demonstrated better glycemic control on inclusion of carbohydrates with low glycemic index as compared to foods with high glycemic index [5-7]. Similarly, consumption of diets with low carbohydrate content are documented to achieve better metabolic profiles including glycemic indices and lipid profiles when compared with equicaloric diet with high carbohydrate content [8-10]. However, the influence of inclusion of sucrose in all daily meals on glycemic indices prior to and after achieving glycemic control in subjects with diabetes has not been examined.

Our study demonstrates that impact of consumption of diet containing sucrose for 4 days on indices of diurnal glycemia as well as HbA1c and fructosamine levels in subjects with new onset type 2 diabetes is dependent on glycemic control. Diurnal dysglycemia is further exacerbated on consumption of diet containing sucrose prior to initiation of therapy at diagnosis of diabetes (Table 2). In contrast, the indices of glycemic control including all diurnal glucose levels as well as hemoglobin A1c and fructosamine concentrations remained unchanged following ingestion of sucrose containing meals on attaining and maintaining desirable glycemic control (Table 2).

The data regarding effect of ingestion of sucrose containing diet on glycemic control is variable in the literature [11-16]. Some previous studies reported deterioration of glycemic control [11-13] while other reports documented no change in metabolic control on consumption of sucrose containing meal in subjects with diabetes mellitus diet [14-16]. However, none of these studies reported degree of glycemic control in participating subjects. Moreover, all these studies examined glucose levels for a few hours following a single meal containing sucrose after an overnight fast in contrast to our protocol of ingestion of all daily meals containing sucrose for 4 days on diurnal glycemia as well as hemoglobin A1c and fructosamine levels. Alternatively, the quantity of sucrose in the experimental diets was variable in individual studies with maximum amount being $23 \%$ of calories provided by carbohydrates [11-16]. In contrast sucrose content was $50 \%$ providing carbohydrate calories in all daily meals in our study. Finally and importantly, none of these studies examined the impact on glycemic indices following consumption of sucrose containing meals for several days in subjects prior to 
and after attaining and maintaining desirable glycemic control. Therefore, data in any of these studies is not comparable to findings in our study.

Deterioration of glycemic indices for ingestion of meals containing sucrose may be attributed to inhibited insulin secretion and paradoxically enhanced glucagon release in response lack of entry of glucose into pancreatic beta and alpha-cells respectively as well as incretin secreting cells due to insulin resistance, the major pathophysiologic mechanism in onset of type 2 diabetes [1,17]. Furthermore, lack of deterioration of glycemic indices following consumption of sucrose containing meals may be attributed to improvement in synthesis and secretion of insulin, glucagon and incretins secondary to enhanced entry of glucose into cells via improvement in both insulin secretion and sensitivity secretion $[1-3,17]$.

\section{Conclusion}

Influence of ingestion of meals containing sucrose on diurnal glycemic pattern as well as hemoglobin A1c and fructosamine concentrations is dependent on degree of glycemic control. It is apparent that ingestion of sucrose may not impair diurnal glycemia in subjects with type 2 diabetes with desirable glycemic control.

\section{Compliance with ethical standards}

\section{Acknowledgments}

Author is grateful to Sarah Exley for assistance in preparation of the manuscript.

\section{Disclosure of conflict of interest}

Author has no conflict of interest.

\section{References}

[1] Kabadi MU, Kabadi UM. Effects of glimepiride on insulin secretion and sensitivity in patients with recently diagnosed type 2 diabetes mellitus. Clin Ther. 2004 Jan; 26(1): 63-9.

[2] Korytkowski M, Thomas A, Reid L, et al. Glimepiride improves both first and second phases of insulin secretion in type 2 diabetes. Diabetes Care. 2002 Sep; 25(9): 1607-11.

[3] Kabadi UM. Major Pathophysiology in Prediabetes and Type 2 Diabetes: Decreased Insulin in Lean and Insulin Resistance in Obese. J Endocr Soc. 2017; 1(6): 742-750.

[4] ADA standard of medical care, diabetes care.

[5] Thomas D, Elliott EJ. Low glycaemic index, or low glycaemic load, diets for diabetes mellitus. Cochrane Database Syst Rev. 2009; (1): CD006296.

[6] Wang Q, Xia W. Zhao Z, Zhang H. Effects comparison between low glycemic index diets and high glycemic index diets on HbA1c and fructosamine for patients with diabetes: A systematic review and meta-analysis. Prim Care Diabetes. 2015; 9(5): 362-9.

[7] Ojo 00, Adebowale F, Wang XH. The Effect of Dietary Glycaemic Index on Glycaemia in Patients with Type 2 Diabetes: A Systematic Review and Meta-Analysis of Randomized Controlled Trials. Nutrients. 19 Mar 2018; 10(3): 373.

[8] Goldenberg JZ, Day A, Brinkworth GD, Sato J, et al. Efficacy and safety of low and very low carbohydrate diets or type 2 diabetes remission: systematic review and meta-analysis of published and unpublished randomized trial data. BMJ. 2021 Jan 13; 372: m4743.

[9] Rosemary Huntriss, Malcolm Campbell, Carol Bedwell. The interpretation and effect of a low-carbohydrate diet in the management of type 2 diabetes: a systematic review and meta-analysis of randomised controlled trials Eur J Clin Nutr. Mar 2018; 72(3): 311-325.

[10] Tay J, Luscombe-Marsh ND, Thompson CH et al. Comparison of low- and high-carbohydrate diets for type 2 diabetes management: a randomized trial. Am J Clin Nutr. Oct 2015; 102(4): 780-90. 
[11] Coulston AM, Hollenbeck CB, Swislocki AL, Chen YD, Reaven GM. Deleterious metabolic effects of highcarbohydrate, sucrose-containing diets in patients with non-insulin-dependent diabetes mellitus. Am J Med. 1987; 82(2): 213-20.

[12] Coulston AM, Hollenbeck CB, Donner CC, Williams R, Chiou YA, Reaven GM. Metabolic effects of added dietary sucrose in individuals with noninsulin-dependent diabetes mellitus (NIDDM). Metabolism. Oct 1985; 34(10): 962-6.

[13] JP Bantle, DC Laine, JW Thomas. Metabolic effects of dietary fructose and sucrose in types I and II diabetic subjects JAMA. 19 Dec 1986; 256(23): 3241-6.

[14] JP Bantle, JE Swanson, W Thomas, DC Laine. Metabolic effects of dietary sucrose in type II diabetic subjects Diabetes Care. Sep 1993; 16(9): 1301-5.

[15] Malerbi DA, Paiva ES, Duarte AL, Wajchenberg BL. Metabolic effects of dietary sucrose and fructose in type II diabetic subjects. Diabetes Care. Nov 1996; 19(11): 1249-56.

[16] Raatz SK, Johnson LK, Picklo MJ. Consumption of Honey, Sucrose, and High-Fructose Corn Syrup Produces Similar Metabolic Effects in Glucose-Tolerant and -Intolerant Individuals. J Nutr. 2015; 145(10): $2265-72$.

[17] Udaya M. Kabadi Diabetes Mellitus: Disorder of Cellular Dysfunction Due to Lack of Entry into Cell of Glucose. The Most Efficient Fuel for Cellular Function* Open Journal of Endocrine and Metabolic Diseases. 31 March $2021 ; 11(3)$. 